Revista Latinoamericana de Medicina Conductual/

Latin American Journal of Behavioral Medicine

volumen 1, número 1, Agosto 2010 -

Enero 2011.

Publicación semestral editada por la

Sociedad Mexicana

de Medicina Conductual, A. C.

RFC:MMC010815817, Cantera 116-1,

Santa Ursula Xitla, Delegación Tlalpan,

México, D. F., C. P. 14420, México.

Editores responsables: María del Rocío

Hernández Pozo, editora@rlmc-mex.org

y Malaquías López Cervantes, mlopez@ rlmc-mex.org.

Página: http://www.journals.unam.mx/ index.php/rlmc

y www.rlmc-mex.org. Reserva de derechos al uso exclusivo número: 04-2010-030511590400-203, ISSN: 2007-0810, ambos otorgados por el Instituto Nacional del Derecho del Autor, Secretaría de Educación Pública, México. Responsable de la última actualización de este número: Alicia Barrera, Unidad de Informática, SMMC, Cantera 116-1, Santa Ursula Xitla, Delegación Tlalpan, México, D.F., C.P. 14420, México. Fecha de la última modificación 23 de Julio del 2010.

Las opiniones expresadas por los autores no necesariamente reflejan la postura del cuerpo editorial en esa materia.

Queda prohibida la reproducción total o parcial del contenido de la revista sin autorización escrita previa de uno de los editores de la revista, o del presidente de la Sociedad Mexicana de Medicina Conductual, A. C.

\section{Revista Latinoamericana de Medicina Conductual / Latin American Journal of Behavioral Medicine}

\section{Editores:}

Ciencias de la Conducta:

María del Rocío Hernández Pozo, FES Iztacala, UNAM, México

Ciencias Médicas:

Malaquías López Cervantes, Facultad de Medicina, UNAM, México

Editores Asociados:

Nazira Calleja Bello, Facultad de Psicología, UNAM, México

Jorge Víquez Rodríguez, FES Iztacala UNAM, México

Everardo J. F. Camacho Gutiérrez, ITESO, México

Ernesto Lorenzo Ravelo Contreras, Universidad Católica de Colombia

Asistente Editorial:

Sandra Cerezo Reséndiz, FES Iztacala UNAM, México

\section{Comité Editorial:}

Alcaraz Víctor Manuel, Universidad Veracruzana, México

Arango Lasprilla Juan Carlos, Virginia Commonwealth University, USA

Arrivilla Quintero Marcela, Pontificia Universidad Javeriana, Cali, Colombia

Balague Gloria, University of Illinois at Chicago, USA

Cobián Mena Alberto, Universidad de Ciencias Médicas de Santiago de Cuba, Cuba

Elder John, School of Public Health, San Diego State University, USA

Feldman de Chaberman Lya, Universidad Simón Bolívar, Venezuela

Flórez Alarcón Luis, Universidad Nacional de Colombia, Colombia

Grau Ábalo Jorge, Instituto Nacional de Oncología y Radiobiología, Cuba

Jinich Horacio, UCSD Medical Center, San Diego, Ca., USA

Juárez González Jorge, Instituto de Neurociencias, CUCBA, Universidad de Guadalajara, México

Kaplan Robert, UCLA, School of Public Health, UCLA, USA

Laham Mirta, Instituto de Psicocardiología, Buenos Aires, Argentina

López Espinosa Antonio, CICAN, Universidad de Guadalajara, México

Lorenzo Ruiz Alexis, Hospital Pediátrico de Tarará, Ciudad de la Habana, Cuba

Mathews Judith R., Medical Center, University of Nebraska, USA

Medina Mora Maria Elena, Instituto Nacional de Psiquiatría Ramón de la Fuente, México

Méndez Ramírez Ignacio, IIMAS, UNAM, México

Oldenburg Brian, Monash University, Australia

Ontiveros Patricia, Hospital del Niño Morelense, Cuernavaca, Mor. México

Ortiz Peralta Juan, Hospital General Cuernavaca, IMSS, México

Ribes Iñesta Emilio, Universidad Veracruzana, México

Rodríguez Ortega Graciela, Facultad de Psicología, UNAM, México

Román Hernández Jorge, Instituto de Salud de los Trabajadores, La Habana, Cuba

Sallis James F., San Diego State University, USA

Spitalnick Josh S., School of Medicine, Emory University, Atlanta, Georgia, USA

Tuomisto Martti T., University of Tampere, Finland

Vinaccia Stefano, Universidad de San Buenaventura, Medellín, Colombia 